J. Synchrotron Rad. (1999). 6, 207-208

\section{In situ XAFS studies of the adsorption of benzene in zeolite beta: differences between fluorescence and electron yield detection}

\author{
Jeroen A. van Bokhoven, ${ }^{a, b^{*}}$ H. Sambe ${ }^{b}$, D.E. \\ Ramaker ${ }^{b}$ and D.C. Koningsberger
}

\author{
"Utrecht University, Somonnelaan 16, $3584 \mathrm{CH}$ Utrecht, \\ The Netheriands, ${ }^{b}$ Chemistry Department, George \\ Washington University, Washington, DC, USA \\ Email:J.A.vanBokhoven@chem.uu.nl
}

By using a newly designed cell to perform in-situ XAFS at the A] $\mathrm{K}$ edge the adsorption of benzene in Beta zeolite is studied. The data were collected both in fluorescence and electron yield mode. The adsorption of benzene changed the near edge structure of the Al $\mathrm{K}$ edge in Beta-zeolite. These changes are much larger when the data are collected in the electron yield mode. A broad satellite peak has been observed in the electron yield data. This peak can be assigned to a core-hole 2-electron final state. The 2 electron final state occurs since in addition to the excited electron another electron is transferred from the benzene to the $3 p$ orbital of the $\mathrm{Al}$ absorber atom (charge transfer, CT). In fluorescence mode this peak is much less pronounced, because the twoelectron state decays primarily via Auger decay.

Keywords: soft X-ray, in-situ AI XAFS, charge transfer shake up, zeolite, fluorescence, electron yield

\section{Introduction}

Zeolites are open pore alumino-silicate structures, which find large application as molecular sieves, catalysts, or catalyst supports (J.B.Nagy et al., 1998). These structures are under extensive investigation for their great economical interest. The relationship between structure and activily is very important in understanding catalytic processes and essential in designing improved catalysts.

By using in-situ XAFS at the Al K edge, the interaction of hydrocarbons (aromatics, olefins and paraffins) with the acid sites in zeolites is studied. The newly designed in-situ XAFS apparatus (ILEXAFS, In-situ Low Energy X-ray Absorption Fine Structure) (van Bokhoven, J.A. et al, 1998) has been used. We find that fluorescence and electron yield detection show remarkable differences in the spectral line shapes. These differences arise as a result of a charge transfer shake-up satellite peak that has much greater intensity in the electron yield mode, because the corehole, two electron final state decays primarily via Auger decay.

This phenomenon makes analysis of the interaction of the adsorbate with the zeolite framework visible. Further, this work points to the importance of collecting both electron yield and fluorescence data, since the differences help to highlight these important satellite features.

\section{Experimental}

The measurements have been performed in the newly designed in-situ apparatus, ILEXAFS, In-situ Low Energy X-ray
Absorption Fine Structure, which has been installed at beamline 3.4 at the SRS Daresbury (UK) (van Bokhoven, J.A. et al, 1998). Fluorescence (using a gas proportional counter) and electron yield (measuring drain current) data have been collected simultaneously.

As synthesised Beta ( $\mathrm{Si} / \mathrm{Al}=11.6)$ is calcined $60^{\circ} \mathrm{C} /$ hour to $450^{\circ} \mathrm{C}$, and kept for 8 hours in order to remove the template. This sample is referred to as H-Beta. The H-Beta is saturated with benzene by flowing He, saturated with benzene, over the zeolite powder at RT. This sample is called Beta-benzene. Samples are pressed into a wafer and no carbon-dilution has been applied. Benzene is chemisorbed at RT and complete filling of the pores is expected (Schaefer, D.J. et al., 1997).

\section{Results and discussion}

The Al $\mathrm{K}$ edge spectra of zeolite H-Beta as detected by electron yield (top) and fluorescence (bottom) are shown in Figure 1. Although the intensity of the white line in the fluorescence spectrum is reduced compared to the electron yield spectrum, due to self-absorption of the fluorescence radiation by the sample, these methods of detection show very similar spectra. Both spectra show the well-known characteristic features of a tetrahedrally coordinated aluminumoxide (van Bokhoven, J.A. et al., 1997), namely the broad $1587 \mathrm{eV}$ peak $(20 \mathrm{eV}$ above the edge), and the sharp white line positioned at $1568 \mathrm{eV}$. The weak features at 1572 and $1577 \mathrm{eV}$ reflect the p-DOS influenced by long range scattering around the absorber (Cabaret et al., 1996) These peaks are indicated by arrows. Howewver, the presence of a small fraction of octahedrally coordinated Al cannot be excluded.

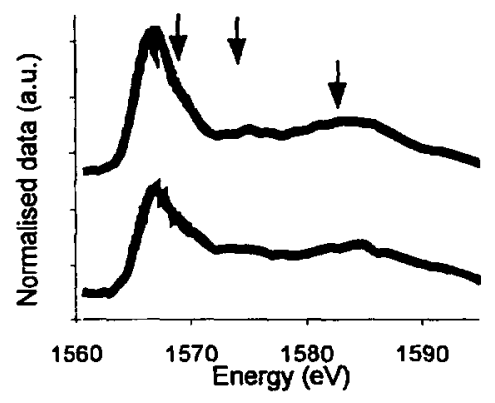

Figure 1. Al K edge of H-Beta as measured by electron yield (top) and fluorescence (bottom).

Figure 2 shows Al $\mathrm{K}$ edge XAS spectra of Beta-benzene as detected by electron yield (top) and fluorescence (bottom). The near edge of Beta-benzene in the electron yield mode is remarkably different from the fluorescence mode. In the Betabenzene as collected by electron yield a broad peak a few eV above the edge is completely dominating the edge. This peak is absent in the H-Beta. This is emphasised in Figure 3, in which the Al $\mathrm{K}$ edge of H-Beta (top) and Beta-benzene (bottom) as collected by electron yield are compared. The broad component just above the edge is absent in the H-Beta.

The fluorescence spectrum of Beta-benzene in Figure 2 (bottom), which has been collected simultaneously with the electron yield, shows a much smaller feature at this position. However, the characteristic features of tetrahedrally coordinated $\mathrm{Al}$ are still present. The $1568 \mathrm{eV}$ white line is now visible as a shoulder in the electron yield detected spectrum (as emphasised 
by the dashed line), and $20 \mathrm{eV}$ above the edge, the shape resonance is still visible. A change in coordination would have resulted in the (near) disappearance of these features.

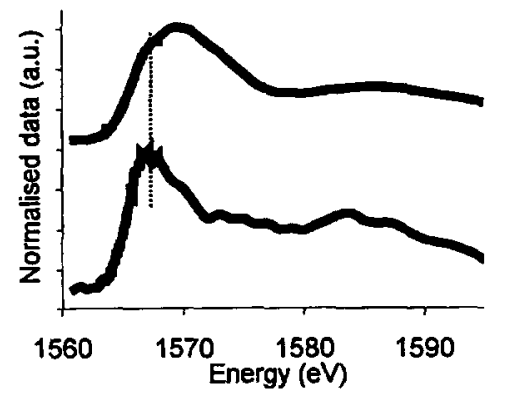

Figure 2. Al $\mathrm{K}$ edge of Beta-benzene as collected by electron yield (top) and fluorescence (bottom).

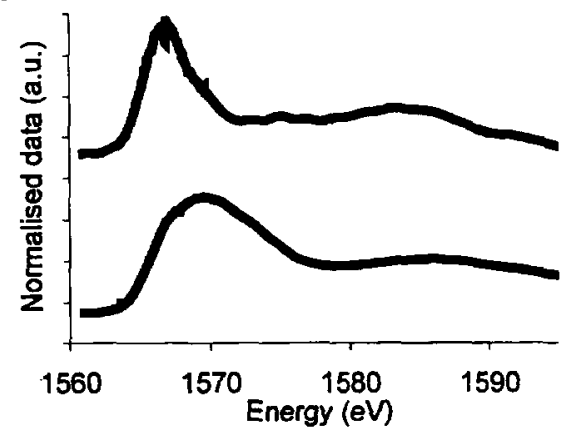

Figure 3. Electron yield spectra of H-Beta (top) and Beta-benzene (bottom).

The new broad feature cannot be attributed to the presence of a coordination other than tetrahedral or to any local geometric distortions. These would have been visible in both electron yield and fluorescence detected spectra. This is further confirmed by ${ }^{27}$ Al MAS NMR spectroscopy, which shows that only tetrahedral $\mathrm{Al}$ is present in Beta-benzene (spectrum not shown).

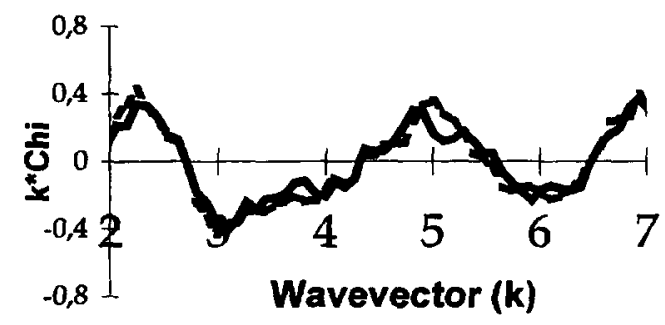

Figure 4. EXAFS of AJ K edge of H-Beta (dotted) and Beta-benzene (solid).

The EXAFS collected on the samples indicate no changes in distance and coordination number within the noise-level. After background subtraction and normalisation of the data, no changes in coordination number and Debye-Waller factor or distance can be seen. This is shown in Figure 4, where fluorescence collected data are presented.

Electron yield detected spectra are surface sensitive (S.M.Heald, 1988), while fluorescence detection probes the bulk of the sample. However, the striking difference in the spectra between electron yield and fluorescence data cannot be explained by the increased surface sensitivity of the electron yield detection. A zeolite is saturated with benzene at room temperature and adsorption does not just take place at the outer surface of the zeolite. A surface effect that could have lead to differences between fluorescence and electron yield is thus unexpected.

We attribute the broad peak in the electron yield spectrum to a charge transfer shake-up process. In electron spectroscopy, such as XPS, shake-up and shake-down processes are well known (E.Stern 1982, G. van der Laan et al., 1986, S. D'Addato et al., 1994). Often these final state effects arise from the creation of charge transfer complexes, which produce in the case of XPS a state at generally higher binding energy when the core electron is excited high into the continuum. The XPS final state then is a core-hole one-electron state. In the XAS case, the photoelectron is excited to localised states just above the edge, where it interacts with the electron that is transferred producing a core-hole twoelectron final state. In the Beta-benzene case, the extra electron is transferred from the benzene $\pi$-system to the Al $3 p$ orbital to help screen the core hole. The electron-electron repulsion between the transferred electron and the photoelectron pushes this state above the absorption edge, which is exactly the case in Beta-benzene as revealed in the electron yield mode.

This satellite state is barely visible in the fluorescence mode. This is due to the preference of the shake-up state to relax via Auger decay. The Auger electron creates many secondary electrons on its way out of the sample, making it highly visible in the electron yield spectrum, compared to the fluorescence yield.

The position of the shake-up peak relative to the edge is a measure of the energy needed for the charge transfer (CT). The intensity is dependent on the nature of the XAS final state and the initial ground state. Preliminary results show that the different aromatic molecules have a somewhat different $\mathrm{CT}$ energy as well as different intensity. The interaction of these molecules with the framework, partly determined by geometric factors and partly by electronic factors, are very important to catalysis. Current research is focused on using this new tool to study the interaction of adsorbates with catalytic surfaces.

\section{Conclusions}

Using the new apparatus, which makes in-situ $\mathrm{Al} \mathrm{K}$ edge XAFS possible, a charge transfer process between aromatic molecules and the zeolitic framework, viz. the Al $3 p$ orbitals, has been observed. This shake-up process causes a broad peak above the absorption edge, which dominates the low energy region of the electron yield spectrum. The charge transfer energy can be determined from the position of the shake-up peak.

This charge transfer satellite causes large differences between the electron yield and fluorescence signals and shows that the two detection methods probe different phenomena. Comparing both spectra helps to identify the presence of charge transfer satellites.

\section{References}

van Bokhoven,J.A. ,Sambe,H., Koningsberger,D.C. \& Ramaker,D.E. (1997), J.Phys. IV France. 7 Colloque C2, Supplément au Journal de Physique III d'Avril 1997, C2-835-840.

van Bokhoven,J.A. van der Eerden,A.M.J., Smith,A.D. \&

Koningsberger,D.C., Vol. 7, this issue.

Cabaret (1996), J. Phys.: Condens. Matter, 8, 3691-3704.

Heald, S.M. (1988), in X-Ray Absorption: Principles, Applications,

Techniques of EXAFS, SEXAFS and XANES, (eds.) Prins, R \&

Koningsberger,D.C., Wiley, Chemical Analysis Vol 92, 87-118.

Nagy,J.B. (1998) Symthesis, characterization and use of zeolitic

microporous materials (1998) DecaGen Ltd., Szeget.

Schaefer,D.J., Favre,D.E., Wilhelm,M., Weigel,S.J. \& Chmelka,B.F. (1997), J.Am.Chem.Soc., 119, 9252-9267.

Stern,E. (1982), Phys. Rev. Let., Vol 49, 48, 1353-1356; van der Laan,G. et al. (1986), Physical Review B, Vol 33, 6, 42534263; D'Addato,S. et al. (1994), Europhysics Letters, 26(2) 85-90. 